International Tinnitus Journal. 2019;23(1):1-5.

\title{
Comparing results from vestibular caloric stimulation and vHIT from a specialised outpatient clinic
}

Jakub Sichnarek ${ }^{1,2}$ Eva Mrazkova ${ }^{1-3}$

Evald Zathurecky ${ }^{1-3}$ Hana Tomaskova ${ }^{1}$

\begin{abstract}
Background: Until recently, vestibular caloric stimulation was the only objective diagnostic method for isolating the function of the lateral semi-circular canals responsible for maintaining balance. Since 2014, the Video Head Impulse Test (vHIT) has been applied in the Czech Republic to investigate the function of all semi-circular canals. Studies conducted so far have shown that the results of these two methods do not often agree.

Methods: The aim of this study was to compare the results of vestibular caloric stimulation and vHIT in a cohort of patients with peripheral vestibular syndrome at a specialized outpatient clinic. The study lasted from July 2016 to August 2017 and included patients with unilateral peripheral vestibular syndrome $(n=32 ; 24$ females and 8 males, mean age 49.4 years) and a positive result with either vestibular caloric stimulation or VHIT.

Findings: $90 \%$ of the patients had a positive vestibular caloric stimulation result, while the VHIT was positive only in $50 \%$ of the patients. Both methods were positive in 13 subjects, vestibular caloric stimulation was positive and vHIT was negative in 16 subjects and in 3 cases vestibular caloric stimulation was negative and vHIT was positive. Based on these numbers, both methods concur in $45 \%$ of the cases. In 13 patients, apart from lateral canal disorders, vHIT revealed disorders in one of the vertical canals - the front right in 4 , the rear right in 2 , the front left in 3 and the rear left in 4. When sorted into subgroups of the "TiTrATE" diagnostic algorithm based on the time course and triggering factor, vHIT was found to be positive for acute spontaneous vertigo in $62.5 \%$ of cases. vHIT was negative for $70 \%$ of chronic and spontaneous episodic complaints.

Conclusions: The diagnosis of peripheral vestibular disorders should take the pathophysiological basis of the disease into account, as this can differ in peripheral disorders. This could then explain the discrepancy between the results of the two methods. The time course of the disease can be a predictor of the vHIT outcome. Localizing the impaired function of particular semi-circular canals using VHIT can inform selective vestibular rehabilitation.
\end{abstract}

Keywords: Vertigo, peripheral vestibular syndrome, vestibule-ocular reflex, vestibular rehabilitation.

${ }^{1}$ Department of Epidemiology and Health Protection Institute, Faculty of Medicine, University of Ostrava, Ostrava, Czech Republic

${ }^{2}$ Department of Hearing and Balance Disorder Centre, Ostrava Poruba, Czech Republic

${ }^{3}$ Department of Otorinolaryngology, Hospital with outpatient clinic Havirov, Czech Republic

*Send correspondence to:

Jakub Sichnarek

Department of Epidemiology and Health Protection Institute, Faculty of Medicine, University of Ostrava, Ostrava, Czech Republic, Email: sicharekj@gmail.com Phone:

$+420739633381$

Paper submitted to the ITJ-EM (Editorial Manager System) on January 30, 2018; and accepted on February 28, 2019. 


\section{INTRODUCTION}

Dizziness is a common health issue, with $20-35 \%$ of the human population experiencing it over their lifetime ${ }^{1,2}$ and it accounts for $3.3-4.4 \%$ of the total number of emergency department visits. In $24-44 \%$ of these cases, the symptoms are due to a peripheral disturbance of the inner ear vestibules ${ }^{3,4}$. It should be noted that positional vertigo (occurring in $10 \%$ of the general population) is a separate phenomenon and we do not deal with it in this paper ${ }^{5}$. Vestibular caloric stimulation has been used for over 100 years mainly due to its clinical applicability and the need for discriminating peripheral vestibular disorders ${ }^{6}$. The thermal gradient formed when water is applied to the external auditory canal is used to monitor the function of the Vestibular of Reflex (VOR) based on the endolymph flux in the lateral semi-circular canal ${ }^{7}$. vHIT is a more modern method of diagnosing the VOR and the function of not only the lateral semi-circular canal, but also the front and rear canals. The principle of examination has remained the same since 1988, but thanks to high-speed video recording of eye movement, it is now possible to objectively quantify the disorders and identify pathological compensatory saccades, which cannot be detected by direct human observation. Therefore, VHIT has a higher sensitivity and specificity compared to clinical $\mathrm{HIT}^{8}$. We have come across a few recent publications that describe discrepancies between the results of vestibular caloric stimulation and the video Head Impulse Test. These may be due either to the time course of the disorder or the different physiological principles on which the two tests are based ${ }^{9-11}$. Curthoys recommends that when considering the methodology, one must keep in mind the fact that the sensitivity and specificity of vHIT relates to the function of semi-circular canals only in cases where dizziness is caused by a known malfunction. The only such cases are those where the vestibular nerve has been surgically disrupted in treating a tumour. When these patients are compared to a set of healthy people, the sensitivity and specificity of VHIT are both 1.0 and the diagnostic accuracy is $100 \%$. Comparisons of vHIT results with results from vestibular caloric stimulation for cases of vertigo due to varied aetiology will only yield a statistical match ${ }^{12,13}$. The incidence of positive $\mathrm{VHIT}$ results increases with acute vertigo ${ }^{9}$. Therefore, it is important, while comparing the results of the two tests, to consider setting where the tests were administered (inpatient or outpatient setting) as the time course of vertigo may vary. Differential diagnosis of vertigo is often quite tricky. At centralized points of admission, it is critical to discriminate between life-threatening conditions and benign symptoms of the disorder. Newman-Toker and Edlow point to the fact that diagnostic algorithms are often outdated by several decades ${ }^{14}$. They propose the new "TiTrATE" diagnostic system, which concentrates on distinguishing vestibular symptoms based on the time course and triggering factor, instead of categorizing the type of dizziness based on a predetermined classification. The system facilitates differential diagnosis of equilibrium disorders by trying to eliminate cases where serious causes of vertigo are not correctly diagnosed ${ }^{15}$. VHIT has long been part of the HINTS algorithm for screening acute states of vertigo in centralized points of admission. The "HINTS" diagnostic system is an extension of "TiTrATE" differential diagnosis ${ }^{13-19}$. In this paper we describe the comparison between the results of vestibular caloric stimulation and vHIT administered in a specialized outpatient clinic.

\section{MATERIALS AND METHODS}

The presented study was conducted in the form of a prospective comparative analysis, and ran from July 2016 to August 2017 in the outpatient facility of the Centre for Hearing and Balance Disorders in Ostrava. All patients were examined by a neurologist based on complaints of vertigo. A detailed medical history of the patients was recorded and they underwent clinical Neurotological examination including objective audiometry, nystagmus monitoring with video nystagmograph record, stabilometric examination, vestibular caloric stimulation and vHIT. A total of 98 individuals were examined, of which a cohort of 32 individuals with unilateral peripheral vestibular syndrome and a positive result with vestibular caloric stimulation or vHIT was selected for statistical analysis. Exclusion criteria for selecting the cohort were as follows central type spontaneous and positional nystagmus, Benign Paroxysmal Positional Vertigo (BPPV) ${ }^{16}$, bilateral vestibular hyporeflexia a lateral difference in vestibular caloric stimulation of less than $25 \%$, fixation index of vestibular caloric stimulation greater than $60 \%$. The vestibular caloric stimulation was conducted using a Hortmann Aquamatic water caloric stimulator in combination with a Video Nystagmograph (VNG) system (ICS Chartr 200 VNG) for binocular movement recording at a $60 \mathrm{~Hz}$ sampling rate. The examination was conducted with the patient lying down on the back with a head-rest giving a $30^{\circ}$ head incline. Each ear was rinsed for 30 seconds with an interval of 5 minutes between each wash. Right side versus left side differences $\geq$ $25 \%$ were regarded as a positive result, i.e., unilateral hyporeflexia. The Fixation Index (FI), or the ability to suppress vestibular nystagmus during the examination, was considered positive at $\geq 60 \%$. The examination was always performed by two healthcare professionals one conducting the caloric testing, the other operating the software. vHIT testing was performed using the GN Otometrics Otosuite V. The distance between the headrest of the patient's chair from the stationary point fixed on the wall was 1 metre. The investigator stood behind the patient's back and carried out a passive rotary motion of the head $\left(5^{\circ}-10^{\circ}\right)$ in the plane of the semi-circular canal at $50-250 \% \mathrm{sec}$ and an acceleration of $750-5000 / \mathrm{sec}^{2}$. When testing the lateral semi-circular canals, the head was rotated in the horizontal plane to the right and left. 
When testing the vertical canal, the head was diverted to the side from the base position by 40 degrees, and then a passive unpredictable vertical upward and downward movement was made. The software received only those head impulses with sufficient speed, acceleration, and the proper plane of the semi-circular canal. The occurrence of corrective saccades (hidden or visible) with a drop in the VOR gain below 0.8 (horizontal canal) and 0.7 (for both vertical canals according to the normative data) was considered a positive result. The examination was always performed by one healthcare professional. The cohort was subsequently sorted into categories according to the "TiTrATE" diagnostic algorithm. This new system focuses on the distribution of vestibular symptoms based on the time course and the trigger factor of the disorder, instead of simply grading the patients and categorizing the type of vertigo. The system aids differential diagnosis of balance disorders and attempts to eliminate "misdiagnosis" of serious causes of vertigo. The cohort included acute spontaneous vestibular syndrome, episodic spontaneous vestibular syndrome, and chronic syndrome ${ }^{14}$. Basic descriptive statistics were used to describe the data. For subsequent analysis, we used the nonparametric paired Wilcoxon signed-rank test and Fisher's exact test. Statistical tests were evaluated at a significance level of $5 \%$, using Stata software, version 13. A signed informed consent form was obtained from all those selected in the cohort, comprising consent to inclusion in a clinical trial.

\section{RESULTS}

In this study included 32 patients ( 24 women, 8 men) with unilateral peripheral vestibular syndrome and were examined in a specialized outpatient clinic. The mean age of the group was $49 \pm 15$ years. Vestibular caloric stimulation was positive in 29 subjects (37\% on the right, $53 \%$ on the left), and negative in three cases (Table 1). VHIT in the lateral channel was negative in 16 cases and positive in 16 cases (Table 2). In 3 cases the defect was restricted to the lateral canal. In all other cases we found a defect also in one of the vertical canals the front right in

Table 1: Results of vestibular caloric stimulation.

\begin{tabular}{cccc}
\hline $\begin{array}{c}\text { Result of caloric } \\
\text { stimulation }\end{array}$ & $\begin{array}{c}\text { Number of } \\
\text { subjects }\end{array}$ & $\%$ & Cumulative \% \\
\hline Negative & 3 & 9.4 & 9.4 \\
Right-lateral hyporeflexia & 12 & 37.5 & 46.9 \\
Left-lateral hyporeflexia & 17 & 53.1 & 100 \\
Total & 32 & 100 & \\
\hline
\end{tabular}

Table 2: Results of the Video Head Impulse Test.

\begin{tabular}{cccc}
\hline vHIT & $\begin{array}{c}\text { Number of } \\
\text { subjects }\end{array}$ & $\%$ & Cumulative \% \\
\hline Negative & 16 & 50 & 50 \\
Positive right & 8 & 25 & 75 \\
Positive left & 8 & 25 & 100 \\
Total & 32 & 100 & \\
\hline
\end{tabular}

4, the rear right in 2, the front left in 3 and the rear left in 4. Comparing results from both the methods, we found that 13 cases showed a concurrent positive result in both vestibular caloric stimulation and VHIT. In 16 others, vestibular caloric stimulation was positive and VHIT was negative; vestibular caloric stimulation was negative and VHIT positive in 3 cases. Based on these values, conformity of the results of both methods is $40.6 \%$. Applying the nonparametric paired Wilcoxon signedrank test, we found a statistically significant difference between the results of the methods $(p=0.0029)$ (Table 3$)$. We further sorted the cohort into categories according to the "TiTrATE" diagnostic algorithm using the time course, the trigger factor of the defect and relationship to the vHIT result (positive and negative). We found no statistically significant difference (Fisher's exact test, $p=0.112$ ). vHIT was positive for acute spontaneous vestibular syndrome in $62.5 \%$ of cases. $25 \%$ of the subjects tested positive for episodic vestibular problems, and $12.5 \%$ of the subjects were positive for chronic conditions (Table 4).

\section{DISCUSSION}

We present a cohort of patients with unilateral peripheral vestibular syndrome who were examined in a specialised outpatient clinic. A positive vHIT result was found in half the examined subjects, but statistically significant concordance in results between vHIT and vestibular caloric stimulation was just above $40 \%$. A positive vHIT result was more frequent in patients with acute dizziness (62.5\%). We did not compare the sensitivity and specificity of calorie testing and VHIT based on the methodological comments in Curthoys ${ }^{12}$. The concordance of the results from the two tests was $40.6 \%$. The causes for such low concordance are varied. It could be due to the distinct physiological nature of the two assays. vHIT stimulation

Table 3: Comparison of the results of vestibular caloric stimulation and vHIT.

\begin{tabular}{cccc}
\hline & & \multicolumn{2}{c}{ Vestibular caloric stimulation } \\
\cline { 3 - 4 } & & Negative & Positive \\
\hline video Head & Negative & 0 & 16 \\
Impulse Test & Positive & 3 & 13 \\
Nonparametric paired Wilcoxon signed-rank test, $\mathrm{p}=0.0029$ \\
\hline
\end{tabular}

Table 4: Relationship between vHIT and the time course and trigger factors of the defects.

\begin{tabular}{ccccc}
\hline \multicolumn{5}{c}{ Time course of vestibular problems } \\
\hline $\begin{array}{c}\text { Video Head } \\
\text { Impulse Test }\end{array}$ & ASVS & ESVS & CHVS & Total \\
\hline $\begin{array}{c}\text { Negative } \\
\text { result }\end{array}$ & $5(31.25 \%)$ & $10(62.50 \%)$ & $1(6.25 \%)$ & $16(100 \%)$ \\
$\begin{array}{c}\text { Positive } \\
\text { result }\end{array}$ & $10(62.50 \%)$ & $4(25.00 \%)$ & $2(12.50 \%)$ & $16(100 \%)$ \\
Total & $15(46.80 \%)$ & $14(43.75 \%)$ & 3 & 32 \\
& & $(9.38 \%)$ & $(100 \%)$
\end{tabular}

Fisher's exact test, $p=0.112$

Abbreviations: ASVS: Acute Spontaneous Vestibular Syndrome; ESVS: Episodic Spontaneous Vestibular Syndrome; CHVS: Chronic Vestibular Syndrome 
of semi-circular canals is a natural stimulus that operates in a frequency range (up to $5 \mathrm{~Hz}$ ) that a person normally encounters in daily life ${ }^{5}$. On the other hand, the nonphysiological vestibular caloric stimulation captures the very low frequency spectrum at $0.003 \mathrm{~Hz}$. Thus, although both methods investigate the same anatomical region, the results measure two different frequency ranges. It is known that the percentage of positive vHIT results, as well as the concordance of results from both tests, increases in cases of acute vertigo9. The most common condition is vestibular neuritis ${ }^{13}$. We were unable to confirm this relationship statistically in our cohort. Nevertheless, we did notice a trend where vHIT was positive in more (63\%) subjects with acute spontaneous dizziness, while the positive result for episodic spontaneous and chronic conditions was only $30 \%$. A similar trend was described by Mahringer and Rambold in their study ${ }^{9}$. The time course of the disease could be a predictor of the vHIT outcome, but we would probably require a larger cohort size in order to confirm this possibility. We believe that a higher percentage of concordance between the tests and positive VHIT results is possible in places where cases of acute vertigo are admitted. Precise differential diagnosis is especially critical in cases of acute vestibular disorders. Significantly, the physicians Newman-Toker and Edlow draw special attention to this problem. They attribute this misdiagnosis of serious conditions to the obsolete diagnostic procedure based on the patient's own assessment of symptom severity. Specifically, they point to the questions asking the patient to describe her/ his subjective perception of the problem based on which a probable diagnosis is assigned; vertigo=vestibular cause, presynaptic condition $=$ cardiac cause, instability=neurological cause, etc. It is not surprising that we can see no correlation here. Therefore, NewmanToker and Edlow propose the new "TiTrATE" diagnostic system, which concentrates on distinguishing vestibular symptoms based on the time course and trigger factor, instead of categorizing the type of dizziness based on a predetermined classification. Depending on the case history, the system enables the clinician to determine follow-up examination(s). The complex "ATTEST" system aids differential diagnosis of equilibrium disorders by trying eliminate cases where serious causes of vertigo are not correctly diagnosed. As of now, this system has not been tested in a clinical trial, but shows highly consistent results as well as lower costs. Given good case history and accurate clinical examination, imaging methods need not be required in all patients ${ }^{13-20}$. In cases of acute spontaneous vertigo of the peripheral type that can be determined by either vestibular caloric stimulation or vHIT, we can recognise the following causes - vestibular neuritis, labyrinthitis (cochlear-vestibular neuritis) ${ }^{13}$ Ménière's disease, and infarction of the labyrinth ${ }^{13}$. The greatest concordance of results is expected in patients with vestibular labyrinthitis and neuritis, as reduced vestibular function remains unchanged over several days or weeks ${ }^{16}$. We expect the lowest concordance in Ménière's disease where enlargement of the membranous duct of the semi-circular canal itself can lead to physiological responses to the rotational stimulation in VHIT. On the other hand, the response in vestibular caloric stimulation is reduced as a result of turbulent flow of endolymph in the enlarged labyrinth. This finding can be significant beyond explaining the discrepancy between the results of both tests. This means that if, in a patient with dizziness, vestibular caloric stimulation is positive and VHIT is negative, dilation of the semi-circular canal duct should be taken into account during diagnosis ${ }^{13}$. Among the causes of spontaneous episodic and chronic dizziness of the peripheral type are Ménière's disease, vestibular migraine, schwannoma of the eighth cranial nerve and autoimmune diseases of the inner ear. Among patients with vestibular migraine, Blödow et al. ${ }^{10}$ found a positive vHIT result in $9 \%$ and a positive vestibular caloric stimulation result in $22 \%$ of the cases. The authors explain this discrepancy by indicating a possible frequency dependence of the two diagnostic methods. The results published by Tranter-Entwistle et al. ${ }^{17}$ show that the diagnosis result depends on the size of the vestibular Schwannoma. In 3 patients in our cohort, the result of vestibular caloric stimulation was negative, while VHIT was positive. This could be due to anatomical variations in the petrous part of the temporal bone, the width of the ear canal, the vascularization and layout of the semi-circular canal itself, or pathology of the middle ear. A final reason could be a false positive from vestibular caloric stimulation, where the individual shows positive caloric stimulation, a negative vHIT but no symptoms of any vestibular disorder ${ }^{16}$. We found isolated defects of the horizontal semi-circular canal only in 3 cases in our cohort. In the other 13 patients, one or both of the ipsilateral vertical canals were defective the front right in 4 , the rear right in 2 , the front left in 3 and the rear left in $4^{17}$. Therefore, vHIT is highly valuable for selective vestibular rehabilitation. The test detects the exact plane of head movement that is not properly interpreted in the CNS and is thus the source of dizziness. Vestibular rehabilitation can thereby target and specifically stimulate the plane of the defective canal which needs to be reconciled to the movement of the head ${ }^{18}$.

\section{CONCLUSION}

When diagnosing peripheral vestibular disorders, it is necessary to take into account the pathophysiological nature of the disease, which differs in peripheral disorders and can cause discrepancy between the results of both the tested methods. The time course of the disease can be a predictor of the outcome in vHIT. The exact localization of defective semi-circular canal function using VHIT provides valuable information for selective vestibular rehabilitation. 


\section{REFERENCES}

1. Agrawal Y, Carey JP, Della Santina CC, Schubert MC, Minor LB. Disorders of Balance and Vestibular Function in US Adults. Archives of Int Med. 2009; 169(10):938-9.

2. Agus S, Benecke H,Thum C, Strupp M. Clinical and Demographic Features of Vertigo: Findings from the REVERT Registry. Front Neurol. 2013; 4:48.

3. Gnerre P, Casati C,Frualdo M, Cavalleri M, Guizzetti S. Management of vertigo from evidence to clinical practice. Italian Journal of Medicine 2015; 9(2):180.

4. Kroenke K, Hoffman RM, Einstadter D. How Common Are Various Causes of Dizziness? A Critical Review. South Med J. 2000; 93(2):160-7.

5. Bárány R. Physiology and pathology of the human's archway clinical studies, 1907.

6. Von Brevern M, Bertholon P, Brandt T, Fife T, Imai T, Nuti D,et al. Benign paroxysmal positional vertigo Diagnostic criteria. J Vestib Res. 2015; 25(4):105-17.

7. Barin K, Jasobson GP, Shepard NT. Background and technique of caloric testing in Balance function assessment and management. $2^{\text {nd }}$ edition. Plural Publishing.2016: 283.

8. Weber KP, Macdougall HG, Halmagyi GH, Curthoys IS. Impulsive Testing of Semicircular Canal Function Using Videooculography. Ann N Y Acad Sci. 2009; 1164(1):486-91.

9. Mahringer A, Rambold HA. Caloric test and video-head-impulse: a study of vertigo/dizziness patients in a community hospital. Eur Arch Oto-Rhino-Laryngol. 2014; 271(3):463-72.

10. Blödow A, Heinze M, Bloching MB, Von Brevern M, Radtke A, Lempert T. Caloric stimulation and video-head impulse testing in Ménière's disease and vestibular migraine. Acta Otolaryngol. 2014; 134(12):1239-44.
11. Zellhuber S,Mahringer A, Rambold HA. Relation of video-headimpulse test and caloric irrigation a study on the recovery in unilateral vestibular neuritis. Eur Arch Oto-Rhino-Laryngol. 2014; $271(9): 2375-83$.

12. Curthoys IS, Jacobson GP, Shepard NT. The video head impulse test (VHIT). Balance function assessment and management. $2^{\text {nd }}$ edition. Plural Publishing. 2016: 419.

13. Halmagyi GM, Chen L, Macdougall HG, Weber KP, Mcgarvie LA, Curthoys IS. The Video Head Impulse Test. Front Neurol. 2017; 8:258-9.

14. Newman-Toker DE, Edlow JA. Titrate: A Novel Evidence-Based Approach to Diagnosing Acute Dizziness and Vertigo. Neurol Clin. 2015; 33(3):577-9.

15. Macdougall HG, Weber KP, Mcgarvie LA, Halmagyi GM, Curthoys IS. The video head impulse test: Diagnostic accuracy in peripheral vestibulopathy. Neurology. 2009; 73(14):1134-41.

16. Manzari L, Burgess AM, Hamish G. Macdougall HG, Bradshaw AP, Curthoys IS. Rapid fluctuations in dynamic semicircular cana function in early Ménière's disease. Eur Arch Oto-Rhino-Laryngol. $2011 ; 268(4): 637-9$.

17. Tranter-Entwistle I, Dawes P, Darlington CL, Smith PF, Cutfield N. Video head impulse in comparison to caloric testing in unilateral vestibular schwannoma. Acta Oto-Laryngolo. 2016; 136(11):111014.

18. Fetter M, Dichgans J. Vestibular neuritis spares the inferior division of the vestibular nerve. Brain. 1996; 119:755-63.

19. Edlow JA, Cone DC. Diagnosing Dizziness: We Are Teaching the Wrong Paradigm. Acad Emerg Med. 2013; 20(10):1064-66.

20. Edlow JA, Gurley KL, Newman-Toker DE. A New Diagnostic Approach to the Adult Patient with Acute Dizziness. J Emerg Med. 2018; 54(4):469-83. 